\title{
Advanced Piezoelectrics: Materials, Devices, and Their Applications
}

\author{
Tao Li, ${ }^{1}$ Jan Ma, ${ }^{1}$ Mohammed Es-Souni, ${ }^{2}$ and Peter Woias ${ }^{3}$ \\ ${ }^{1}$ School of Materials Science and Engineering, Nanyang Technological University, Singapore 639798 \\ ${ }^{2}$ Institute for Materials and Surface Technology, University of Applied Sciences Kiel, 24149 Kiel, Germany \\ ${ }^{3}$ Department of Microsystems Engineering, IMTEK, University of Freiburg, 79110 Freiburg, Germany
}

Correspondence should be addressed to Tao Li, tli@ntu.edu.sg

Received 18 March 2012; Accepted 18 March 2012

Copyright (C) 2012 Tao Li et al. This is an open access article distributed under the Creative Commons Attribution License, which permits unrestricted use, distribution, and reproduction in any medium, provided the original work is properly cited.

After decades of research and development, piezoelectric materials have been applied in a wide range of applications, ranging from household appliances to industrial equipments. In areas such as precision and acoustic engineering, piezoelectric material is well accepted to be one of the leading functional materials. Today, due to the high demand, particularly from the electronic, energy, and biomedical industries, researchers are driven to the exploration of new materials and device configurations for new applications. At the same time, engineers are making continuous effort to improve the existing technologies. As the scope of the area is vast, it is necessary to provide an overview on different branches of piezoelectric materials. We would like to thank all the authors who submitted their papers to this special issue. We also hope that this special issue can give an insight on the current research and development status of piezoelectric materials community. We further expect that this issue could stimulate the generation of new ideas and technologies for advanced future applications.

This special issue accepted sixteen papers from industries, research institutes, and universities worldwide. The topics touched include failure analysis, thin film, nanotechnologies, materials synthesis, fabrication technique, modeling, nondestructive testing, sensors, actuators, transducers, ultrasonics, cleaning, vibration suppression, energy harvesting, and characterization techniques.

In the paper "Local fatigue evaluation in PZT thin films with nanoparticles by piezoresponse force microscopy," a PZT film was fabricated and the local switching polarization behavior of the film during fatigue was investigated using scanning piezoelectric microscopy. It was found that the introducing of barium titanate (BT) nanoseeds has significantly improved the fatigue performance.

In the paper "Redirection of Lamb waves for structural health monitoring," Lamb wave based structural health monitoring was proposed and its benefits were explored. This technique can potentially be used to improve the detectability of defects in difficult-to-inspect locations.

In the paper "A surface acoustic wave ethanol sensor with zinc oxide nanorods," a surface acoustic wave ethanol sensor using $\mathrm{ZnO}$ nanorods was developed and tested. It has shown that the nanorod structure can increase the frequency shift of the sensor.

In the paper "Damping analyses of structural vibrations and shunted piezoelectric transducers," an experimental method was used to calculate the damping energy in a mechanical system. Both piezoelectric damping and structural damping were analyzed. This method is meaningful for vibration suppression and control.

In the paper "Static electric force and measurement principle of material constants in electrostrictive material," a model regarding the electric force acting on the material and method to measure the electrostrictive coefficients were proposed. Factors affecting the measurement accuracy were also discussed based on the proposed model.

In the paper "Electromechanical and dynamic characterization of in-house-fabricated amplified piezo actuator," a diamond-shaped amplified piezo actuator was fabricated and tested. Essential parameters such as displacement and block force were measured.

In the paper "Fabrication of simple and ring-type piezo actuators and their characterization," the fabrication and 
characterization of a piezoelectric multilayer structure were illustrated. The procedure was introduced in detail. The obtained actuator can be used for fluid flow control in space vehicles.

In the paper "Modeling hysteresis with inertial-dependent Prandtl-Ishlinskii model in wide-band frequency-operated piezoelectric actuator," the hysteresis and nonlinear effect of a piezoelectric actuator was modeled and characterized. The results are useful for enhancing the controlling capability of an actuator.

In the paper "Comparison of analog and digital self-powered systems in multimodal vibration suppression," the methods of digital and analog vibration suppression were proposed and compared. It shows that both methods are effective in improving damping performance. Also, the self-powering feature makes the methods useful in various applications.

In the paper "Active vibration control of a microactuator for the hard disk drive using self-sensing actuation," a microactuator was combined with sensing function, which can then be used for hard disk vibration control. The work has also demonstrated that the method is effective in suppressing the vibrational modes.

In the paper "Infra-through ultrasonic piezoelectric acoustic vector sensor particle rejection system," an ultrasonic cleaning system was developed to remove the dust from a sensor filament. Therefore, it helps to improve the cleanness and hence maintain the measurement accuracy of the sensor.

In the paper "Solid state adaptive rotor using postbuckled precompressed, bending-twist coupled piezoelectric actuator elements," a piezoelectric actuator was developed, which can be integrated on a solid stator adaptive helicopter rotor. This method is promising on the performance enhancement of the helicopter by reducing part count, increasing reliability, and allowing for decoupled individual blade control of rotor blades.

In the paper "Synthesis and characterization of $\mathrm{Pb}\left(\mathrm{Zr}_{0.53} \mathrm{Ti}_{0.47}\right) \mathrm{O}_{3}-\mathrm{Pb}\left(\mathrm{Nb}_{1 / 3} \mathrm{Zn}_{2 / 3}\right) \mathrm{O}_{3}$ thin film cantilevers for energy harvesting applications," a PZT-PZN thin film cantilever was fabricated for the purpose of energy harvesting. The dielectric properties of the film were characterized. The generated electric signal of the cantilever harvester was studied and simulated under various conditions.

In the paper "Epitaxial piezoelectric $\mathrm{Pb}\left(\mathrm{Zr}_{0.2} \mathrm{Ti}_{0.8}\right) \mathrm{O}_{3}$ thin films on silicon for energy harvesting devices," PZT thin film was fabricated. Its microstructure and electrical properties were investigated. As an energy harvester, it was compared with other devices and its energy harvesting potential was examined.

In the paper "Time-dependent piezo-electro-magnetoelastic fracture behavior of conducting cracks in a half-space under anti-plane mechanical and in-plane electric and magnetic impact," piezo-electro-magneto-elastic materials under transient loads were modeled and discussed. One of the important findings in this paper is that there exists a strong coupling between stress and electrical and magnetic field near crack tips.

In the paper "Exact solution for an anti-plane interface crack between two dissimilar magneto-electro-elastic half-spaces," the fracture behavior of a piezo-electro-magneto-elastic composite was modeled. Fourier transform was applied. Some important parameters, such as electric displacement and magnetic induction, were determined and discussed.

Tao $\mathrm{Li}$

Jan Ma

Mohammed Es-Souni

Peter Woias 

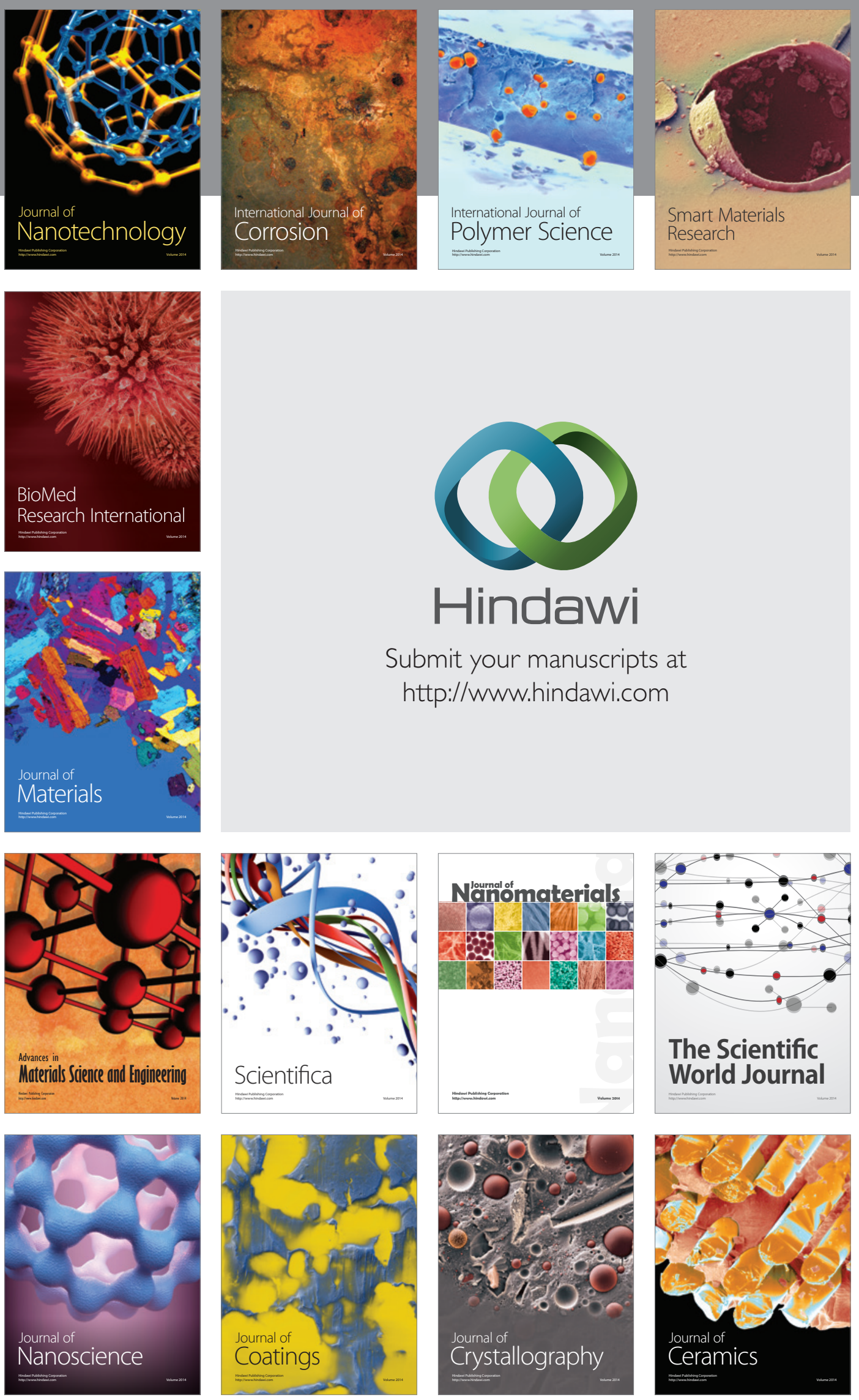

The Scientific World Journal

Submit your manuscripts at

http://www.hindawi.com

\section{World Journal}

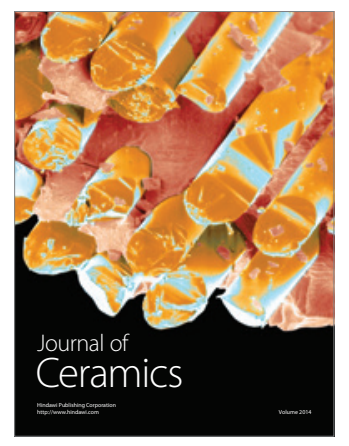

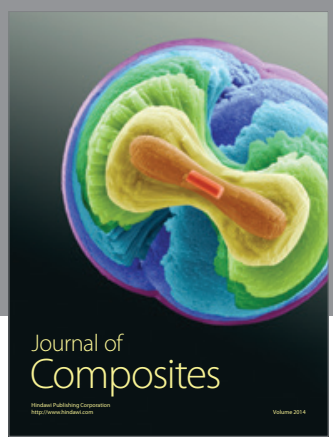
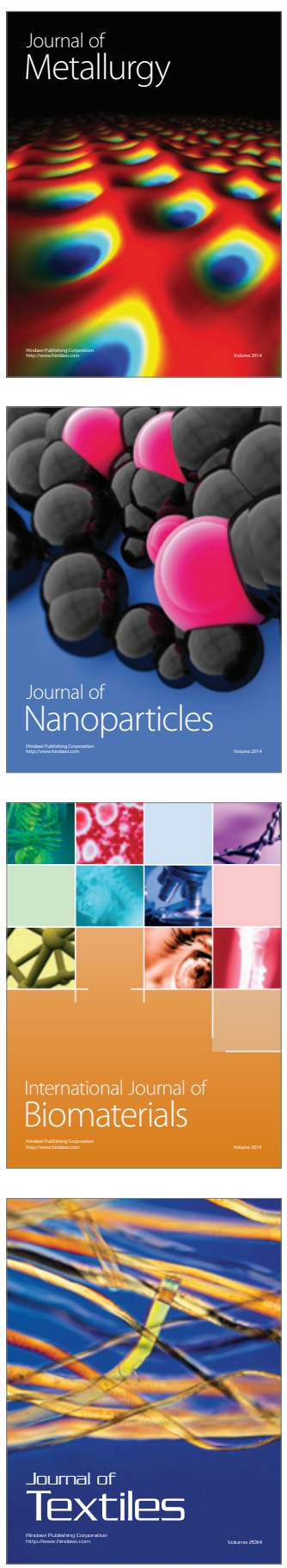